\title{
Active Antenna Tracking System with Directional Antennas for Enhancing Wireless Communication Capabilities of a Networked Robotic System
}

\author{
Byung-Cheol Min* \\ The Robotics Institute, Carnegie Mellon University, Pittsburgh, Pennsylvania 15213 \\ e-mail: bmin@cs.cmu.edu
}

Eric T. Matson

M2M Lab., Department of Computer and Information Technology, Purdue University, West Lafayette, Indiana, 47907; Department of Computer Science and Engineering, Dongguk University, Seoul 100-715, Republic of Korea

e-mail: ematson@purdue.edu

\section{Jin-Woo Jung}

Department of Computer Science and Engineering, Dongguk University, Seoul 100-715, Republic of Korea

e-mail: jwjung@dongguk.edu

Received 14 June 2014; accepted 22 February 2015

\begin{abstract}
This paper presents a networked robotic system design capable of enhancing wireless communication capabilities (communication range and bandwidth). The core of the system is active antenna tracking with directional antennas. The proposed system is decentralized and consists mainly of a mobile robot system and a command center system. Each system is equipped with off-the-shelf network devices such as antennas, access points (AP), and network switches. For directional antennas to be beneficial to our system, we propose a weighted centroid algorithm (WCA) to provide active antenna tracking and direction-of-arrival (DOA) estimation. This system can be used in GPS-denied environments as our system does not require the aid of additional sensors to provide location information. Through extensive field experiments in different environments, including a fire training center and with various antenna selections, such as omni-to-omni, omni-to-directional, and directional-to-directional antennas, we demonstrate the effectiveness of our proposed system. We expect that our system can be applied in a variety of rescue, surveillance, and emergency scenarios where high bandwidth and long-distance communications are needed. (C) 2015 Wiley Periodicals, Inc.
\end{abstract}

\section{INTRODUCTION}

As robots gradually replace manpower in the fields of safety, security, and rescue, communication quality between robots is becoming a significant issue that needs to be fully addressed. In many cases, rescue robots need to be deployed distantly from a command center to carry out their missions. For example, in the event of fire, like the Illinois fire in July 2013 (See Figure 1), long-distance and high-quality communication is useful in enabling firefighters to control a firefighting robot remotely from a safe zone. High-quality communication is needed to view high-definition images transmitted from the robot. If the robot is not equipped

${ }^{*}$ Corresponding author; this work was done when he was affiliated with Purdue University. An earlier version of this paper was presented at the 2013 IEEE International Symposium on Safety, Security, and Rescue Robotics (SSRR), Sweden, 2013, and was published in its Proceedings, available at http:/ /ieeexplore.ieee.org/xpls/abs_all.jsp?arnumber $=6719347$ with such communication capabilities, human operators may have to stay in areas of very high temperature or with noxious fumes in order to maintain connectivity with the robot. To avoid such hazardous situations, high-bandwidth and long-range wireless communication technology is required.

In this paper, we directly focus on such problems and develop a robotic system capable of enhancing wireless communication capabilities. First, to achieve distant-range communications, we used directional antennas. For directional antennas to be beneficial in our system, we proposed a weighted centroid algorithm (WCA), which is a method for active antenna tracking, and direction-of-arrival (DOA) estimation. These methods are designed to maintain the best network quality between a mobile robot and a command center by a precision tracking capability without the aid of location information sensors such as the global positioning system (GPS) and inertial measurement unit (IMU).

In addition, we adopted WiFi network technology that allows for the provision of a broadband network 


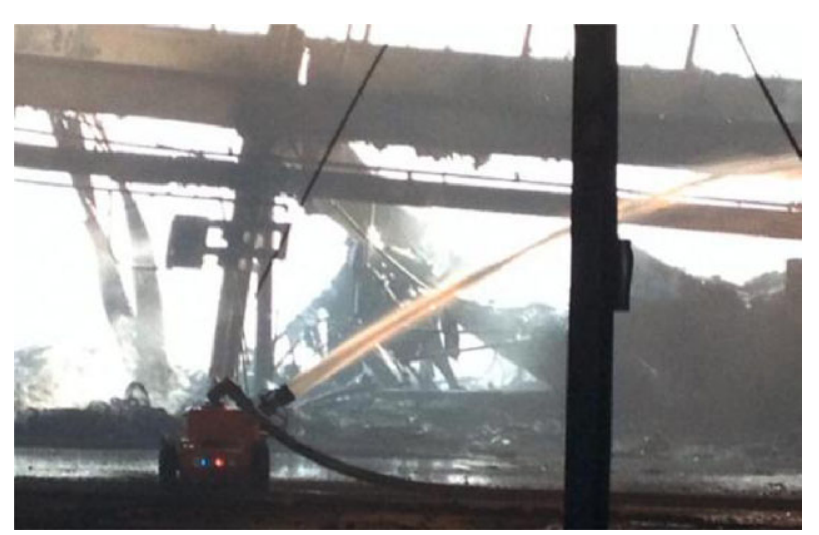

Figure 1. A firefighting robot fights a working tire fire in Illinois, USA (Min, Matson, \& Khaday, 2013b).

capable of transferring far higher throughput compared to conventional communication techniques such as IR (infrared), Bluetooth, and ZigBee communications (Lee, $\mathrm{Su}$, \& Shen, 2007; Shahzad \& Oelmann, 2014; Mahmood et al., 2015). Because of this higher throughput, high-definition images can be transferred, which will greatly increase the versatility of the robot for surveillance. In addition, secured communication can be easily established through encryption and Service Set Identification (SSID) technology.

We also proposed using off-the-shelf network devices such as access points (AP) and network switches to create a broadband network between a robot and the command center. As our system is fully decentralized, and only requires the single robot and the command, it can minimize complexity and cost, which is shown in the section on related studies that required the use of multiple robots. We believe that our proposed system can be applied to a variety of rescue, surveillance, and emergency scenarios where highbandwidth and distant-range wireless communications are needed.

The remainder of this paper is organized as follows. In Section 2, we present an overview of related studies on robotic communication and DOA estimation. In Section 3, we introduce methods for active antenna tracking and DOA estimation for directional antennas beneficial to robotic communications. We then detail components of the complete system in Section 4. In Section 5, we describe the setup and results of field experiments to verify the performance of the proposed system. Section 6 summarizes the conclusions and future scope of this work.

\section{RELATED STUDIES}

\subsection{Robotic Communication}

There have been a number of previous attempts to improve the network performance of robotics in applications such as disasters and emergencies where long-range communications are needed. Most of those attempts employ multiple robots having wireless networking capabilities to achieve the improvement.

For human existence detection in case of disasters, Tuna, Gungor, \& Gulez (2014) proposed an autonomous wireless sensor network deployment system. As the authors were concerned about the connectivity issue, they introduced a role-based exploration approach for cooperative exploration, composed of explorer and relay robot units. Tekdas, Kumar, Isler, \& Janardan (2012) studied the problem of building a commutation bridge between a signal source and a destination with mobile robots. From this research, they showed that multiple mobile robotic hubs could provide connectivity service in applications such as disaster response. Hsieh, Cowley, Kumar, \& Taylor (2008) presented an experimental study to maintain end-to-end communication links for tasks such as surveillance and reconnaissance, where team connectivity is required for situational awareness. To establish mobile wireless mesh networks and increase network throughput, Nguyen et al. (2012) employed multiple mobile robots. By placing one robot at the end node, i.e., by reducing the hop count required for network traffic to transit through, they could increase network throughput. Pezeshkian, Nguyen, \& Burmeister (2007) proposed an unmanned ground vehicle radio relay deployment system that employs mobile robots that carry multiple relay radio to maintain robust communications. Specifically, the system was designed to have long-range and non-line-ofsight (NLOS) operational capabilities.

All of the research mentioned above has demonstrated the possibility on improving network performance in the robotics domain, but all of these have to employ multiple robots, not a single robot, to fulfill their objectives. For that reason, it is unavoidable that the entire system becomes more complex and expensive.

\subsection{Direction-of-arrival Estimation}

For wireless mobile robot communications, omnidirectional antennas have been typically used. The main advantage of these antennas lies in that they are very easy to install. Due to their spherical radiation pattern, they can be easily mounted anywhere on the robot's body. Also, due to this pattern, they provide a wide coverage area from their center. This efficacy allows multiple clients diffused around the antennas to access wireless communications. Therefore, omnidirectional antennas are often considered to be suitable for communications in a multirobot system.

Whereas omnidirectional antennas provide a wide coverage area, they cannot deliver long communication distances. Also, it is known that omnidirectional antennas are more subject to interference from other signals due to their radiation patterns (Yi, Pei, \& Kalyanaraman, 2003). Recently, to address such problems, the use of directional antennas has received increased attention (Everett, Duarte, Dick, \& 
Sabharwal, 2011; Dai, Ng, Li, \& Wu, 2013). First, long communication distances can be achieved by diverting the RF energy in a particular direction with directional antennas. Second, with a narrower radiation pattern than that of the omnidirectional antenna, the directional antenna can avoid the region where wireless signal congestion occurs. However, because of the narrower radiation pattern, fine-tuning is necessary in order for the antenna to be oriented in a specific angle and direction. Moreover, when the directional antenna is mounted on a moving robot, the orientation of the two directional antennas - i.e., the one installed on the robot and the other at the command center - needs to be continuously adjusted to maintain the communication link and to provide high-quality communications.

For such reasons, a decent method of DOA estimation or direction finding of radio emission sources should be developed for wireless robot systems.

There has been active research in DOA estimation and source localization methods using directional or omnidirectional antennas. Sayrafian-Pour and Kaspar (2006) showed that a receiver equipped with a circular array antenna with beam-forming capability could generate the spatial spectrum of the received power by electronically rotating the main lobe around the $360^{\circ}$ field of view. As a result, the rotating receiver could estimate the position of the transmitter to within a few meters. However, this method requires an array antenna that contributes to an expensive and complicated system, and as such, is unsuitable with our desire to build a cost- and computationally effective system.

Hood and Barooah (2011) described a customized directional antenna with an actuated reflector for estimating DOA of a radio signal. With a simple implementation, they obtained mean errors of less than $4^{\circ}$. Elnahrawy, AustenFrancisco, and Martin (2007) presented a localization approach based on the use of rotational directional antennas and a Bayesian network. For DOA estimation, the authors collected the raw data at $10^{\circ}$ intervals, applied a smoothing function with the average of each point along with its six closest neighbors, and constructed a cosine function from the smoothed curve. Although these methods showed accurate estimation using directional antennas, they have to conduct multiple measurements from multiple devices or over a long period to gather more sample data to produce a better estimation. Because of the multiple measurements, it was very slow and expensive to process their estimation methods.

Graefenstein, Albert, Biber, and Schilling (2009) proposed a new method to obtain the relative DOA between the mobile robot and the static node using commodity rotatable radio hardware. Since their methods require prerecording a reference radiation pattern, it would not be possible to deal with dynamic environments and unknown interference.

Kim and Chong $(2007,2008)$ conducted DOA estimation using the ratio of radio signal strength from a dualdirectional antenna system to guide mobile robots to a target and docking system. Although they showed that the robot could arrive at the target position by following estimated DOAs, the maximum range between the robot and the target position was only about $20 \mathrm{~m}$. As the range or area of interest for this paper is much larger than that range, it is not applicable to our research.

\section{ANTENNA TRACKING}

\subsection{Active Antenna Tracking}

If two directional antennas in a point-to-point network are operated in a completely open and perfectly known location, it would not be difficult to determine the necessary orientations for the best connection with the aid of GPS and IMU (Min, Lewis, Matson, \& Smith, 2013a). In such a situation, having the two antennas point at each other would usually provide the best quality of wireless communications. However, this approach is only feasible when both communication sides are equipped with very accurate GPS and a compass sensor (Bapna, Rollins, Foessel, \& Whittaker, 1998). Furthermore, as it is almost impossible to obtain GPS signals in indoor environments, the location functionality cannot be used in environments where directional antennas have the potential to increase wireless capacity (Min et al., 2013a). In addition, in a situation where the effects of multipath and the presence of other wireless interference exist, pointing at each other may not be the best orientation nor guarantee the best quality. Therefore, optimizing the function of the two antennas only by sharing information on their current orientations and positions might be the inadequate approach.

In addition, in a situation where the effects of multipath and the presence of other wireless interference exist, it is hard to predict or calculate the best orientation for a directional antenna without adequate data regarding their effects.

For that reason, this paper proposes an active antenna tracking system and DOA estimation for the self-orientation of directional antennas. First, the proposed system requires two directional antennas mounted on a pan-tilt servo device on each side; i.e., a total of four antennas are used for building a point-to-point network, as depicted in Figure 2.

Figure 2 illustrates the configuration of this system, with the robot on the left side and the command center on the right side. The top antenna on both sides is the actual one for data transmission, so these antennas are paired together. The bottom antenna is for DOA estimation with the opposite side. By rotating the bottom antenna, taking RSSI (radio signal strength indication) measurements and finding the direction with the strongest RSSI from the top antenna on the opposite side, it can compute the best orientation of the top antenna. Therefore, the orientation of each of the top antennas is adjusted periodically by the bottom antennas in each rotation. This active antenna tracking system runs independently on each side, so it might take some time to 


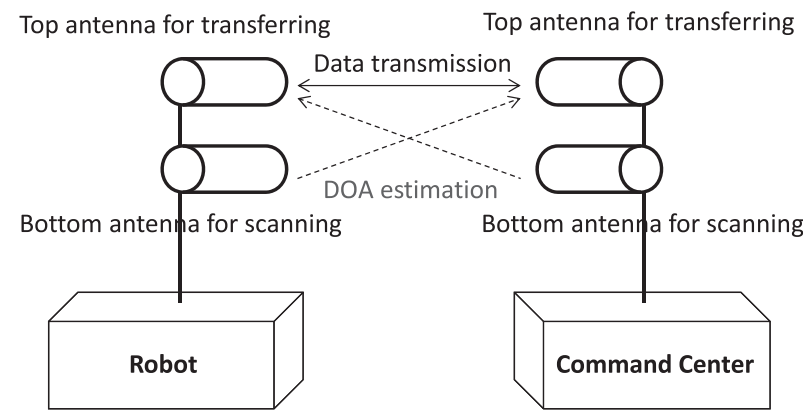

Figure 2. A configuration of the proposed system. The system composed of two directional antennas on each side, so a total of four antennas are installed.

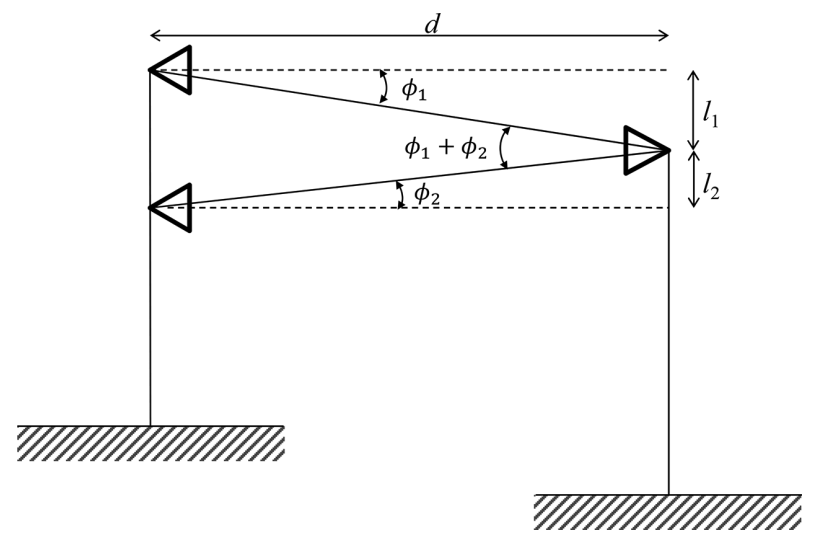

Figure 3. A simplified geometrical model of two wireless links.

adjust the top antenna orientation and to reach the best orientation. Nonetheless, with this approach, realized through the measurement of radio signal strengths, the orientations of the two top antennas can be optimized without the aid of GPS and a compass sensor.

Assuming the two communication sides are far enough apart, and the two antennas are installed on the same vertical axis close to each other, fields of view from the antennas can be projected to almost the same area. To show feasibility of this configuration, a simplified geometrical model is depicted in Figure 3.

In Figure 3, $d$ is a distance between the two sides, $\phi_{1}$ is an angle generated by the height difference between the top two antennas, and $\phi_{2}$ is an angle generated by the height difference between the bottom antenna and the top antenna on the opposite side. Thus, we can obtain

$$
\phi_{1}+\phi_{2}=\tan ^{-1}\left(\frac{l_{1}}{d}\right)+\tan ^{-1}\left(\frac{l_{2}}{d}\right) .
$$

Assuming $l_{1}$ and $l_{2}$ are constant while $d$ varies, the angle difference $\phi_{1}+\phi_{2}$ becomes smaller as $d$ increases in Eq. (1). As this configuration is aimed at the long-range communication, $d$ can be considered to be a very large number,

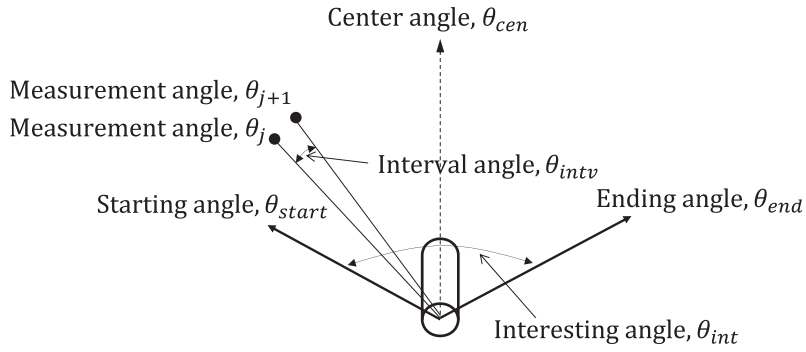

Bottom antenna

Figure 4. Defined parameters for DOA estimation with a directional antenna, when scanning clockwise.

and therefore, the angle difference becomes negligible in the long run.

\subsection{Weighted Centroid Algorithm}

It is known that the measurement of radio signal strengths often contains measurement noise as well as fading caused by the effects of multipath or interference from other electronics devices. Because of this unreliable measurement, estimating the right DOA is difficult. To cope with this, we develop a DOA estimation technique using directional antennas, or WCA, a type of weighted centroid approach. Weighted centroid approaches have been adopted by several research groups (Behnke \& Timmermann, 2008; Blumenthal, Grossmann, Golatowski, \& Timmermann, 2007; Pivato, Palopoli, \& Petri, 2011; Wang, Urriza, Han, \& Cabric, 2011). The previous studies used the distance as the weighting factor through power measured from multiple anchor nodes. In this paper, we examine the directionality of the radiation pattern with a stand-alone directional antenna for DOA estimation. As the basic concept of using weights to obtain the centroid of a data set is similar to the previous studies, we recommend referring to the papers referenced above for a more detailed explanation of the concept of weighted centroid approaches.

Before introducing the WCA, we first define several parameters needed in WCA, as shown in Figure 4, and detailed parameters are described in Table I. From the center

Table I. Setting of parameters.

\begin{tabular}{ll} 
Variables & \multicolumn{1}{c}{ Description } \\
\hline$\theta_{\text {int }}$ & Interesting range where a scanning task performs \\
$\theta_{\text {start }}$ & Starting angle where to start the interesting range \\
$\theta_{\text {end }}$ & Ending angle where to end the interesting range \\
$\theta_{\text {cen }}$ & $\begin{array}{c}\text { Center angle between the staring angle and the } \\
\text { ending angle }\end{array}$ \\
$\theta_{\text {intv }}$ & Interval angle of measurement \\
$\theta_{j}$ & Measurement angle \\
\hline
\end{tabular}




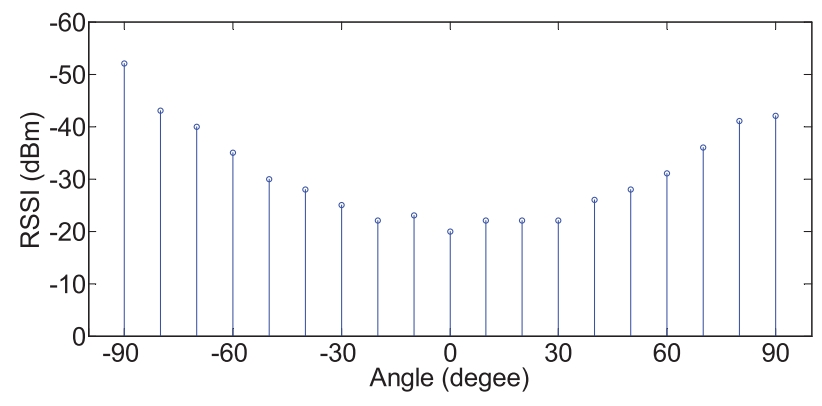

Figure 5. An example of measured signal strength with a rotating directional antenna. The horizontal axis is the measurement angle and the vertical axis is the measured signal strength, RSSI.

of the antenna's body, we define an interesting range $\theta_{\text {int }}$, where a scanning task is performed. Then, the starting angle $\theta_{\text {start }}$, where the range starts; the ending angle $\theta_{\text {end }}$, where the range ends, going either clockwise or counterclockwise from the starting angle in turn, the center angle $\theta_{c e n}$ between the starting angle and the ending angle; and the interval angle of measurement $\theta_{\text {intv }}$ are defined. At the beginning of scanning, the center angle is in front of the device. While scanning from the starting angle to the ending angle, $N_{t}$ times of the measurement task are performed at a measurement angle $\theta_{j}$, where $j$ is the index of the measurement such that $j \in\left\{1,2, \ldots, N_{t}\right\}$, producing $R S S I_{j}$, the measured RSSI at the $j$ th measurement. For the interval angle $\theta_{i n t v}$, it is assumed that this angle can be computed by dividing the interesting range by the total number of measurements $N_{t}$.

Figure 5 shows an example of a measured RSSI from an experiment that was conducted indoors, with a rotary directional antenna, showing the parameters above. In this figure, it is shown that $\theta_{\text {int }}=180^{\circ}, \theta_{\text {start }}=-90^{\circ}, \theta_{\text {end }}=90^{\circ}$, $N_{t}=19$, and therefore, $\theta_{\text {intv }}=10^{\circ}$.

In the first step of the WCA, a single rotary directional antenna measures the signal strength by rotating from $\theta_{\text {start }}$ to $\theta_{\text {end }}$ and produces a set of $R S S I_{j}$. In the second step, a weight is computed by the measured signal strengths at $\theta_{j}$ using the following expression:

$$
w_{j}=10^{\left(\frac{R S S I_{j}}{\gamma}\right)},
$$

where $\gamma$ is a positive gain that should be appropriately determined in every application scenario so that stronger signal strengths are more weighted than weaker signal strengths. Then, the DOA can be estimated by means of weighted centroid approaches as follows,

$$
\widetilde{\Theta}=\frac{\sum_{j=1}^{N_{t}} w_{j} \theta_{j}}{\sum_{j=1}^{N_{t}} w_{j}} .
$$

If we use the measured RSSI shown in Figure 5 again and depict all variables used in Eq. (3) in polar coordinates, it should look like Figure 6 . Here, $\gamma$ was set to 10 , the

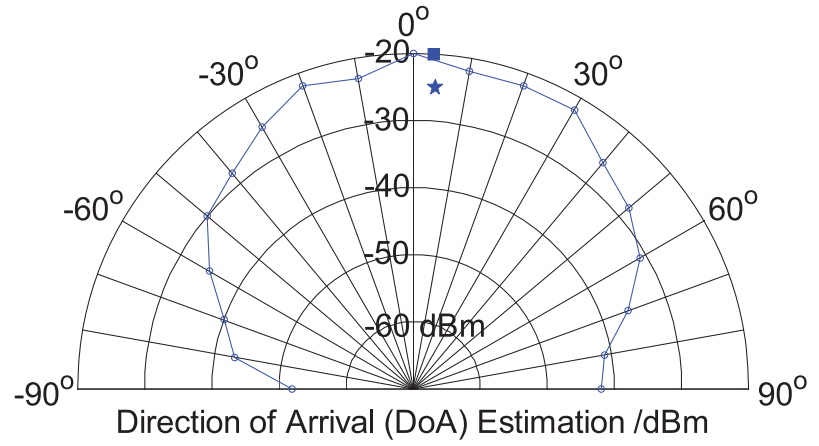

Figure 6. Weighted centroid algorithm (WCA) in a polar coordinate frame.

estimated DOA $\widetilde{\Theta}$ using the WCA was depicted with a symbol " $\star$ " (See nearby $0^{\circ}$ on the angle axis between $-20 \mathrm{dBm}$ and $-30 \mathrm{dBm}$ ) in a polar coordinate, and the actual angle $\widehat{\Theta}$ was depicted with a symbol " $\mathbf{\square}$ ". Note that one can read the estimated DOA and the actual angle in Figure 6 by observing a coordinate of the symbols on the angle axis. Because we do not deal with a distance-related estimation in this paper, we do not need to observe a coordinate of the symbols on the $\mathrm{dBm}$-axis.

With Eqs. (2) and (3), the measured data with strong signal strengths are depicted further from the center in Figure 6, and their angle values become more important to determine the weighted centroid. Conversely, weaker signal strengths are rarely weighted because of the log scale. Therefore, the measured data with weak signal strengths are depicted closer to the center, and their angle values become less important. As a result, it can be said that Eq. (3) calculates a reasonable DOA by averaging the measured data with appropriate weighting.

In fact, as stated in Behnke and Timmermann (2008), weighted centroid approaches have entailed poor estimation when the actual DOAs approached both ends. Considering cases where an actual DOA is near an extreme, the other sample data will necessarily pull the average toward the side opposite the DOA. Thus, even if all sample data are averaged with appropriately assigned weights, an estimated DOA is always pulled toward the side where the most samples reside. In other words, all sample data on the side opposite the side with more data prevent the estimation from approaching the end where an actual DOA dwells.

We have partially modified the WCA to cope with this unavoidable problem. First, this modification is activated after obtaining an estimated DOA $\widetilde{\Theta}$ using the procedure stated earlier. The key modification is that of changing the interesting range $\theta_{i n t}$ with the previously calculated DOA so that the center angle $\theta_{c e n}$ of the range can be placed exactly on the previous DOA. By doing so, this modification can place an actual DOA away from an extreme end in the next scanning, therefore preventing the issues shown earlier. 
To show a feasibility of this modification, we take into account a case where a receiving antenna and a transmitting antenna are static, i.e., they are fixed at a certain location. By using Friis Free-space Equation (Min et al., 2013a) and assuming that the antenna gain of the receiving antenna $G_{r}$ is proportional to $\cos ^{2} \theta$, where $\theta$ is a pointing error, i.e., an angle between the current orientations of the receiving and the transmitting antennas, we can write the power $P_{r}$ in $\mathrm{dB}$ at the receiving antenna as follows,

$$
\left.P_{r}\right|_{\mathrm{dB}}=\left.P_{t}\right|_{\mathrm{dB}}+\left.G_{t}\right|_{\mathrm{dB}}-\left.L_{f}\right|_{\mathrm{dB}}+\left.G_{r}\right|_{\mathrm{dB}} \cos ^{2} \theta,
$$

where $P_{t}$ is output power of the transmitting antenna, $G_{t}$ is the gain of the transmitting antenna, $L_{f}$ is the free-space loss given as $(4 \pi d / \lambda)$, where $\lambda$ is the wavelength and $d$ is the distance between the antennas. Then, we can rewrite the measured signal strength at $\theta_{j}$ as

$$
R S S I_{j}=P_{r_{j}}=K+G_{r} \cos ^{2} \theta_{j}
$$

where $K$ is $\left.P_{t}\right|_{\mathrm{dB}}+\left.G_{t}\right|_{\mathrm{dB}}-\left.L_{f}\right|_{\mathrm{dB}}$, and we assume that it is constant in this verification. Note that we omitted the decibel unit $\left.\right|_{\mathrm{dB}}$ from all variables in Eq. (5) for an easy description.

If the total number of measurement $N_{t}$ is odd and represented as $2 N+1$, then we can rewrite $j_{\text {th }}$ measurement angle at the $k_{t h}$ scanning as follows,

$$
\theta_{j}(k)=\theta_{c e n}(k)+\frac{j \theta_{i n t}}{2 N}
$$

where $(j \in \mathbb{Z} ;-N \leq j \leq N)$.

Then, the next center angle, $\theta_{c e n}(k+1)$ can be obtained by adding the estimated amount of change of DOA with Eqs. (3) and (5) to the current center angle $\theta_{c e n}(k)$ as follows,

$$
\theta_{c e n}(k+1)=\theta_{c e n}(k)+\frac{\theta_{i n t}}{2 N} \frac{\sum_{j=-N}^{N} j 10 \frac{G_{r} \cos ^{2}\left(\theta_{c e n}(k)-\theta_{c e n}{ }^{*}+\frac{j \theta_{i n t}}{2 N}\right)}{\gamma}}{\sum_{j=-N}^{N} 10^{\frac{G_{r} \cos ^{2}\left(\theta_{c e n}(k)-\theta_{c e n} *+\frac{j \theta_{i n t}}{2 N}\right)}{\gamma}}},
$$

where $\theta_{c e n}{ }^{*}$ is the actual DOA that the center angle should be placed by iterations. It is worth noting that a constant $K$ in Eq. (5) has been eliminated in Eq. (7) as it appears in the exponent. tain

By subtracting $\theta_{c e n}{ }^{*}$ from both sides in Eq. (7), we ob-

$$
\begin{aligned}
\theta_{c e n}(k+1)- & \theta_{c e n}{ }^{*}=\theta_{c e n}(k)-\theta_{c e n}{ }^{*} \\
+ & \frac{\theta_{i n t}}{2 N} \frac{\sum_{j=-N}^{N} j 10 \frac{{ }_{r} \cos ^{2}\left(\theta_{c e n}(k)-\theta_{c e n}{ }^{*}+\frac{j \theta_{i n t}}{2 N}\right)}{\gamma}}{\sum_{j=-N}^{N} 10 \frac{G_{r} \cos ^{2}\left(\theta_{c e n}(k)-\theta_{c e n}{ }^{*}+\frac{j \theta_{i n t}}{2 N}\right)}{\gamma}}
\end{aligned}
$$

$$
e(k+1)=e(k)+\frac{\theta_{i n t}}{2 N} \frac{\sum_{j=-N}^{N} j 10^{\frac{G_{r} \cos ^{2}\left(e(k)+\frac{j \theta_{i n t}}{2 N}\right)}{\gamma}}}{\sum_{j=-N}^{N} 10^{\frac{G_{r} \cos ^{2}\left(e(k)+\frac{j \theta_{i n t}}{2 N}\right)}{\gamma}}},
$$

where $e(k)=\theta_{\text {cen }}(k)-\theta_{c e n}{ }^{*}$, i.e., $e(k)$ is the error between the estimated DOA at the $k_{t h}$ scanning and the actual DOA.

If we define $f(e(k))$ as the differentiation of $e(k)$ in discrete time, then

$$
f(e(k))=\frac{e(k+1)-e(k)}{(k+1)-k}=\frac{\theta_{i n t}}{2 N} \frac{\sum_{j=-N}^{N} j 10^{\frac{G_{r} \cos ^{2}\left(e(k)+\frac{j \theta_{i n t}}{2 N}\right)}{\gamma}}}{\sum_{j=-N}^{N} 10^{\frac{G_{r} \cos ^{2}\left(e(k)+\frac{j \theta_{i n t}}{2 N}\right)}{\gamma}}} .
$$

To show the feasibility of modified WCA for the estimated of DOA, we restrict the range of $\theta_{\text {int }}$ to nearly (but less than) $\pi$ at the maximum, and we will show $f(e(k))$ in Eq. (10) goes to zero as the time $k$ increases, graphically.

Figure 7 is the graph of $f(x)$ when the constant $K, G_{r}$, and $\gamma$ are set to the actual values we use for the experiments.

By Eqs. (9) and (10), we can obtain

$$
e(k+1)=e(k)+f(e(k)) .
$$

Equation (11) indicates that the error at the $(k+1)_{t h}$ scanning is determined by the addition of $f(e(k))$ to the current error at the $k_{t h}$ scanning.

As illustrated in Figure 7(a) and (b), the absolute error at the $(k+1)_{t h}$ scanning is always less than the absolute error at the $k_{t h}$ scanning, because $f(e(k))>0$ in the negative domain $e(k) \in\left[-\frac{\pi}{2}, 0\right], f(e(k))<0$ in the positive domain $e(k) \in\left[0, \frac{\pi}{2}\right]$, and $f(e(k))=0$ at $e(k)=0$. Moreover, as shown in Figure 7(a), the absolute value of $f(x)$ is always less than the absolute value of $y=-2 x$. If it was greater than the absolute value of $y=-2 x$, a divergence would take place.

Figure 7(b) shows an enlarged graph of $f(x)$ with a setting of $\gamma$ to 1 that shows a dramatic case where the absolute value of $f(x)$ is sometimes greater than the absolute value of $y=-x$. For instance, the absolute value of $f(e(k+2))$ is greater than the absolute value of $y=-x$ at $e(k+2)$. Because of that, the next $(k+3)_{t h}$ scanning takes place in the opposite domain. Nonetheless, the absolute error at the next scanning, $e(k+4)$ is, of course, less than the absolute error at the $(k+3)_{t h}$ scanning. Therefore, $f(e(k))$ goes to zero as the time $k$ increases and the modified WCA method can always find DOA.

Also, a moving average is used to smooth and thus minimize variations of estimated DOA. This may cause delayed antenna tracking, but it can be minimized by appropriately determining the window size in the moving average. In addition, as the beamwidth of the antennas we use for this research is wide enough to maintain the connection with the opposite antenna, a short delay is acceptable. 


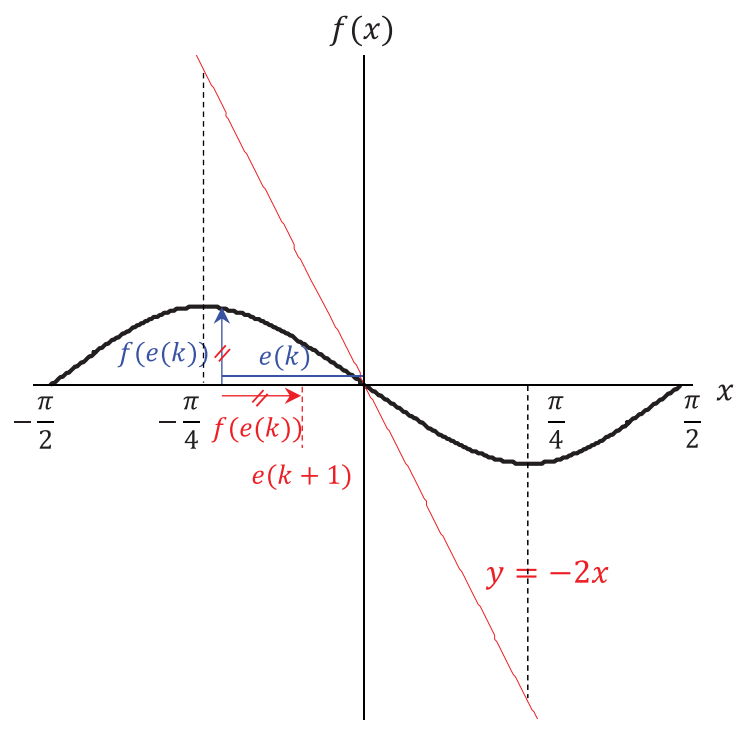

(a) $\gamma=10$

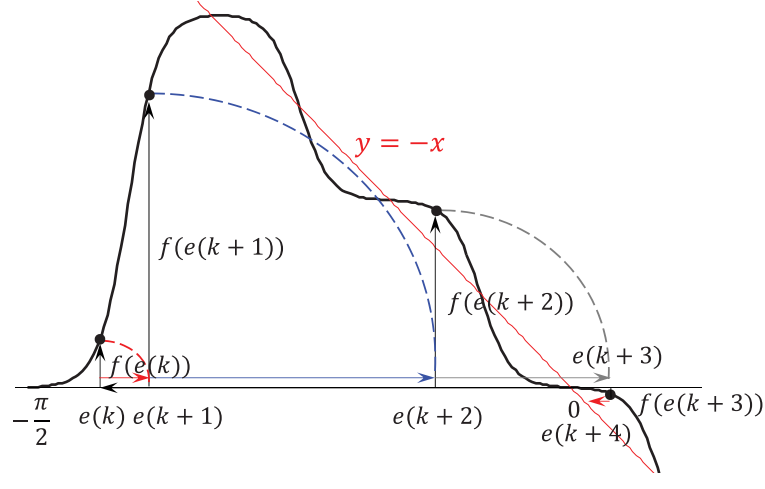

(b) $\gamma=1$

Figure 7. Verification of the modified WCA. (a) Shows that the absolute value of $f(x)$ is always less than the absolute value of $y=-2 x$. Therefore, as $k$ increases, $f(x)$ goes to zero. (b) Shows an enlarged graph with a setting of $\gamma$ to 1 for more detailed explanations of the verification.

\section{DESIGN OF ROBOTIC COMMUNICATION SYSTEM 4.1. Robot System Design}

To test the proposed methods, we have developed a prototype of the robotic system as shown in Figure 8. The complete system mainly consists of a mobile robot system and a

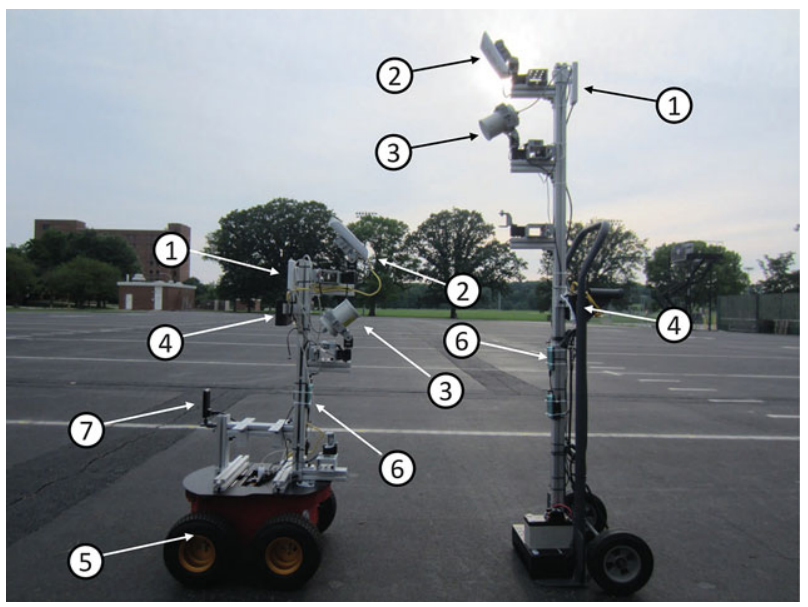

Figure 8. Robotic communication system, composed of the robot system (left) and the command center system (right) - 1) PicoStation wireless AP, 2) NanoStation wireless AP, 3) PCTEL yagi antenna, 4) network switch, 5) P3AT mobile robot, 6) WiFi USB adapter, 7) IP camera. command center system. The mobile robot system is made up of a P3AT mobile robot, a laptop, two APs running with an omnidirectional antenna and a directional antenna, respectively, a yagi antenna, a network switch, a WiFi USB adapter, an internet protocol (IP) camera, and three pantilt servo devices. The command center system is equipped with almost the same components as the robot, but it does not have the P3AT or the IP camera.

\subsubsection{Networking Devices}

Our system is designed to enhance wireless network capabilities by means of antenna tracking with directional antennas that build a point-to-point broadband network. Actually, it is possible to establish the point-to-point network with one of the following three antenna selections: 1) omni-to-omni antennas, 2) omni-to-directional antennas, and 3) directional-to-directional antennas. Hence, we test all of the three antenna selections in this paper and analyze their performance to validate our proposed system.

For the first selection, requiring an omnidirectional antenna on each side, we use a state-of-the-art, low-cost, high-performance, and small wireless AP, PicoStation M2$H P$, manufactured by Ubiquiti Networks Inc. This AP is equipped with a 5-dBi omnidirectional antenna, and supports passive Power over Ethernet (PoE), so it does not require an additional power code. Also, it runs with IEEE $802.11 \mathrm{~g}$ protocol having an operating frequency of $2.4 \mathrm{GHz}$ and produces up to $28 \mathrm{dBm}$ output power. As this device 
was designed to be deployed in either indoor or outdoor environments, it is ideal for applications requiring mediumrange performance and a minimal installation footprint.

For the third selection, requiring two directional antennas on each side, we installed another wireless AP, NanoStation loco $M$, manufactured by Ubiquiti Networks Inc. This $\mathrm{AP}$ is equipped with an 8-dBi directional antenna, which can be seen on the top of the system. Hence, this antenna is used for data transmission. This system also runs with $2.4 \mathrm{GHz}$, and produces up to $23 \mathrm{dBm}$ output power. The beamwidth of this antenna is $60^{\circ}$ at $1 / 2$ power for horizontal and vertical planes. This device was specifically designed for outdoor point-to-point bridging applications. In addition to this NanoStation AP, we installed a small and light yagi antenna, manufactured by PCTEL for DOA estimation. This device can be seen on the bottom of the system. This device has $10 \mathrm{dBi}$ of gain, uses $2.4 \mathrm{GHz}$ frequency range, and has $55^{\circ}$ horizontal and vertical beamwidth at $1 / 2$ power.

For the second selection, requiring an omnidirectional antenna on the robot side and two directional antennas on the command center side, we use a PicoStation AP introduced in the first selection as the omnidirectional antenna. For directional antennas, we use the NanoStation AP and PCTEL yagi antennas introduced in the third selection.

We use a passive PoE managed network switch, TOUGHSwitch, manufactured by Ubiquiti Networks Inc., in order to power the devices that can be powered through PoE, such as two of Ubiquiti's APs and a camera. Also, by using a network switch in the communication system, we can easily add additional network devices or laptops to the established communication link between the robot and the command center. Furthermore, we can utilize this switch when we want to extend wireless signals on the robot side by turning on the PicoStation AP and setting it in a repeater mode. That is, WiFi signals transported through the top directional antenna can be propagated with the omnidirectional antenna.

\subsubsection{Robot Platform}

The P3AT is a four-wheel driven autonomous ground vehicle, developed by Adept MobileRobots. This robot has been widely adopted for research purposes, as it is sturdy and durable and provides open source codes. We also adopted this robot as our mobile robot platform for this research.

\subsubsection{Additional Devices}

We use an Asus Eee laptop, running Linux, to manage highlevel motion planning for the P3AT, to receive radio signal from the Alfa WiFi USB adaptor connected with the bottom directional antenna, and to process DOA estimation.

We have developed a pan-tilt device with off-the-shelf DC servos, manufactured by Robotis Co. Three pan-tilt devices, controlled by an ATMEL128 microprocessor, are installed at each communication side - the first is for the NanoStation AP, the second is for the yagi antenna, and the third is for a digital camera. The motion of the third pan-tilt device is synchronized with the top one so that we can see the current field of view from the top antenna for test purposes. If images from the camera contain a physical body on the opposite side at the center, we could say that our proposed methods work well.

The mobile robot system is also equipped with an IP camera, aircamMini, manufactured by Ubiquiti Networks Inc., as shown in Figure 9(a). This camera is powered through PoE, includes a microphone and has a 1MP/HDTV $720 \mathrm{p}$ resolution and 30 FPS maximum frame rate, so it is suitable for surveillance purposes by being installed on the mobile robot. An example of the snapshot showing a screen taken by this camera and a robot control interface are shown in Figure 9(b).

\subsection{System Architecture}

Figure 10 shows an overview of the robot system architecture.

The laptop is connected by an RJ-45 cable to the PoE network switch, by a serial connection to the P3AT, two pan-tilt devices, and the Alfa USB adapter. A pan-tilt device allows the directional antenna to be oriented in a specific angle autonomously. In this paper, we employ a pan angle only since the directional antenna we chose for this project has about $55^{\circ}$ beamwidth vertically, and therefore, there are few cases where our robot is deployed out of the range. However, it should be noted that vertical beamwidth would also affect wireless communication in some cases.

The PoE network switch, powered by the battery and transformer, provides the power to the APs and IP camera and enables all of the network devices to be connected on the same network.

\section{EXPERIMENTS}

To test the proposed system, we conducted extensive field experiments in four different environments ${ }^{1}$ and with the three different antenna selections stated in Section 4.1.1. For a comparison of the performance of each antenna selection, we implemented a data throughput test while a robot moves away from the command center to its destination. This was done to reinforce the assumption that the strongest wireless signal has a direct correlation to the best signal for a data link connection. To perform this test, the Linux "iperf" command was used to measure a small data transfer over the established link between the robot and the command center. A laptop on the robot side running iperf was set to a server

\footnotetext{
${ }^{1}$ Videos demonstrating the field experiments can be found at https:/ / www.youtube.com/playlist?list=PLqZFsOngJCPNbclekt BoCTd3qVJFYQULw.
} 


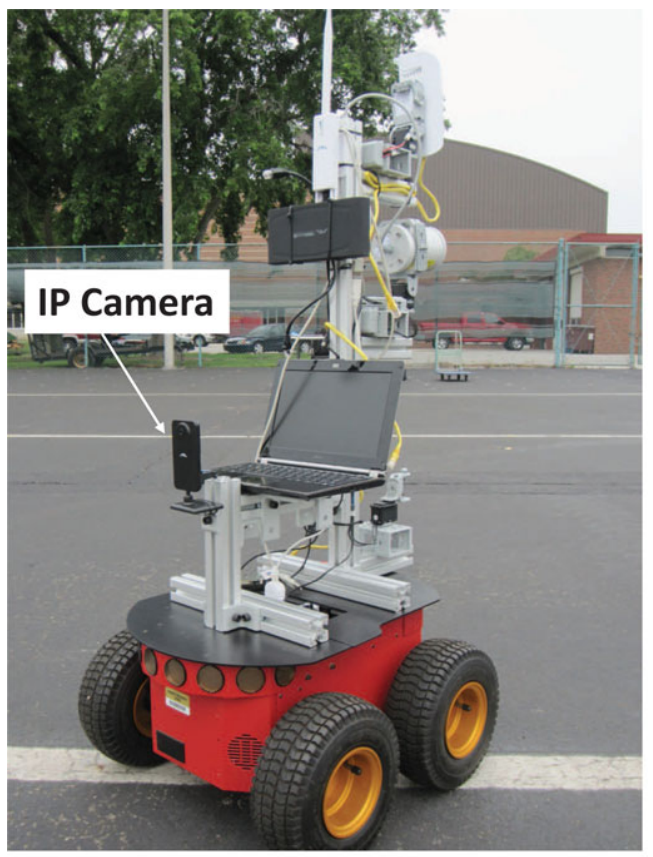

(a)

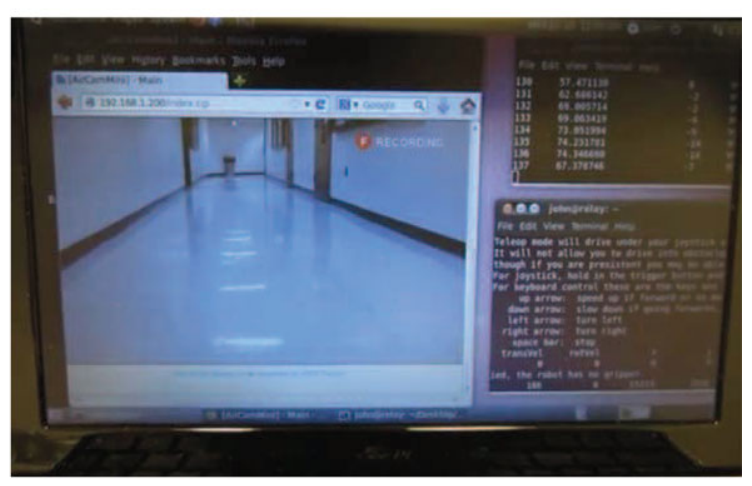

(b)

Figure 9. Mobile robot equipped with an IP camera. (a) IP Camera. (b) Screen.

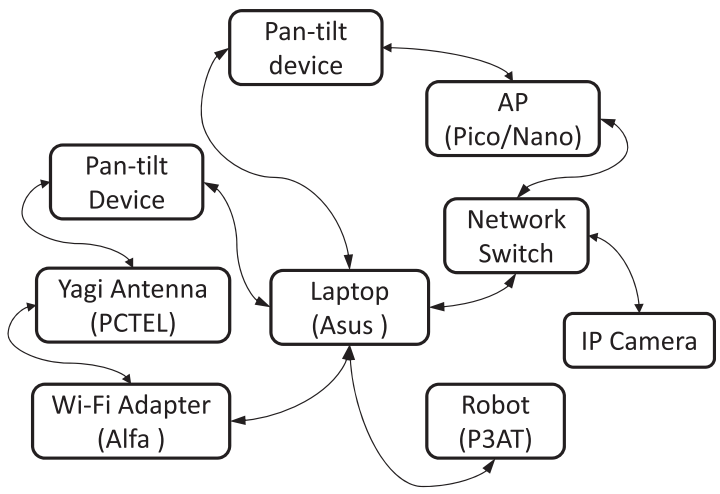

Figure 10. An overview of the communication system architecture: robot system side.

mode, and a laptop on the command center side was set to a client mode. A small amount of data were transferred through the autonomously created link and a measurement of the time-to-transfer rate was performed by iperf. The resulting measurement gives an accurate available throughput for the established link. Since our tracking system only takes into account RSSI, or received signal power, and not packet quality, we can use this test to verify received data integrity, which is especially important for a multipath link.

For experiments with a fair evaluation, each setting was run through at least three different trials. Also, the powers of the two antennas for data transmission, PicoStation and
NanoStation APs, were set to $8 \mathrm{dbm}$ and $5 \mathrm{dbm}$ for the first experiment on range/distance tests so that the total radio signal power can be the same setting of $13 \mathrm{dBm}$. For the three other experiments, we set $13 \mathrm{dBm}$ and $10 \mathrm{dBm}$ to PicoStation and NanoStation APs, respectively.

\subsection{Range/Distance Tests}

We first conducted a field test to show how much improvement we obtain in terms of range/distance with the proposed system. During this test, the robot was manually controlled to move along a long path with an almost constant speed of $0.5 \mathrm{~m} / \mathrm{s}$, as shown in Figure 11.

Figure 12 shows results of the averaged throughput with a standard deviation versus the distance that the robot traveled. As shown in Figure 12, when the robot was equipped with either of the first two antenna selections, it reached up to around $150 \mathrm{~m}$ from the command center and throughput decreased steeply. On the other hand, when the robot was equipped with the third antenna selection, it could reach to $250 \mathrm{~m}$. In fact, the robot could have moved to much further distances, but due to the limited space of the environment, we had to stop at the $250 \mathrm{~m}$. With this third selection, throughput was able to be kept with almost the same values of the initial measurement until the robot reached to $100 \mathrm{~m}$ and decreased gradually, resulting in above $15 \mathrm{Mbps}$ at the final location. From this test, we could show that the third antenna selection significantly outperformed the 


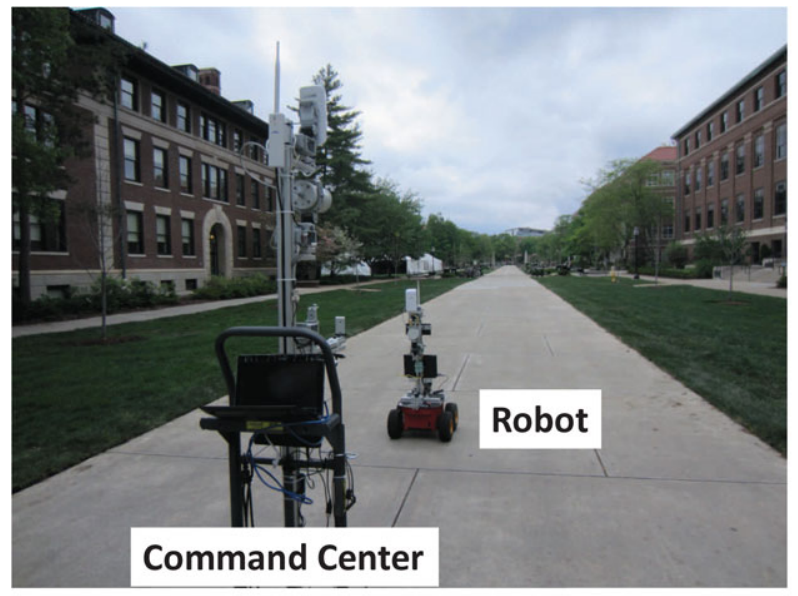

(a)

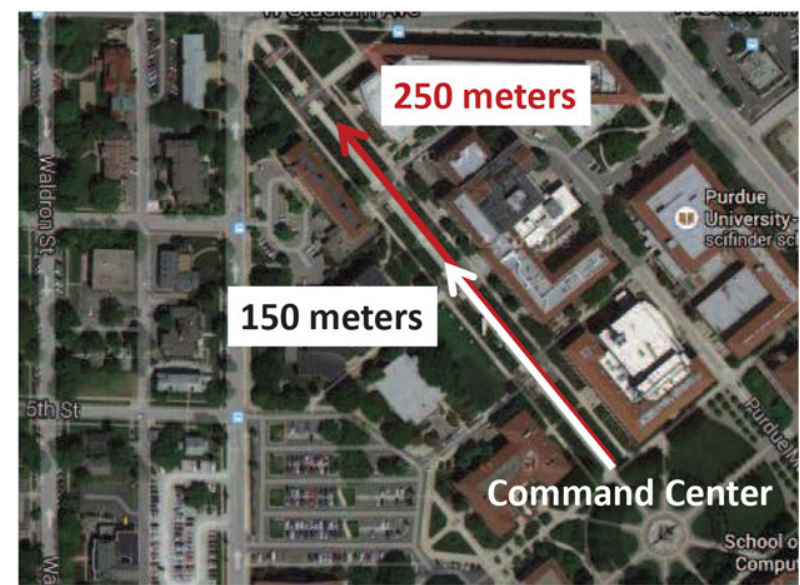

(b)

Figure 11. Range/distance test at Purdue main campus. (a) Purdue Mall. (b) Traveled distances.

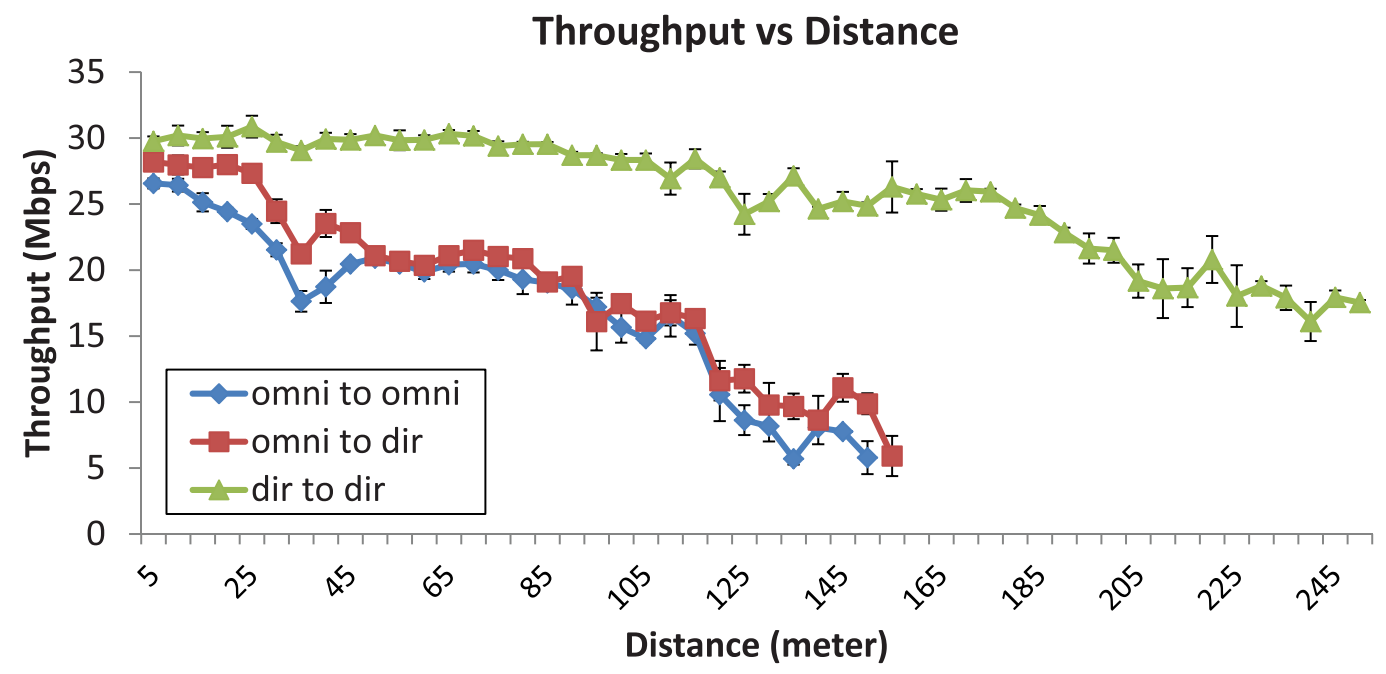

Figure 12. Throughput versus distance test - throughput was measured while the robot was moving along a long path.

other two antenna selections in both range/distance and throughput measurements.

\subsection{Outdoor Test in Open Environments}

For the different environments, we first chose the Purdue marching band practice field whose size is almost the same as a typical football field. This environment was chosen to test cases where the moving robot has to be deployed in an open and outdoor environment and where a long distance and a high quality of communication are required. The environment is shown in Figure 13(a). During this test, the robot was set to move along a designated path with an almost constant speed of $0.9 \mathrm{~m} / \mathrm{s}$. The designated path is shown with a red line on the bottom of Figure 13(b). The total traveling distance of this path is approximately
$130 \mathrm{~m}$ and the longest distance between the command center and the robot is approximately $100 \mathrm{~m}$. For WCA, $\gamma$ was set to 10 , and $\theta_{i n t}$ was set to $100^{\circ}$, resulting in the initial scan performed at $\theta_{\text {start }}=-50^{\circ}, \theta_{\text {end }}=50^{\circ} . N_{t}$ was approximately 25 for most of the tests. These settings were applied to all of the environments.

Figure 14 shows the averaged throughput with a standard deviation for all tests with each antenna selection. As expected, the directional-to-directional antennas, the third selection, outperformed the other two selections by showing far higher throughput by as much as one and a half times. Specifically, the third selection shows very stable data throughput over distance and time. This result indicates that the pair of directional antennas were adjusted and aligned well while the robot was moving. In other words, it validates that our antenna tracking system worked successfully. 


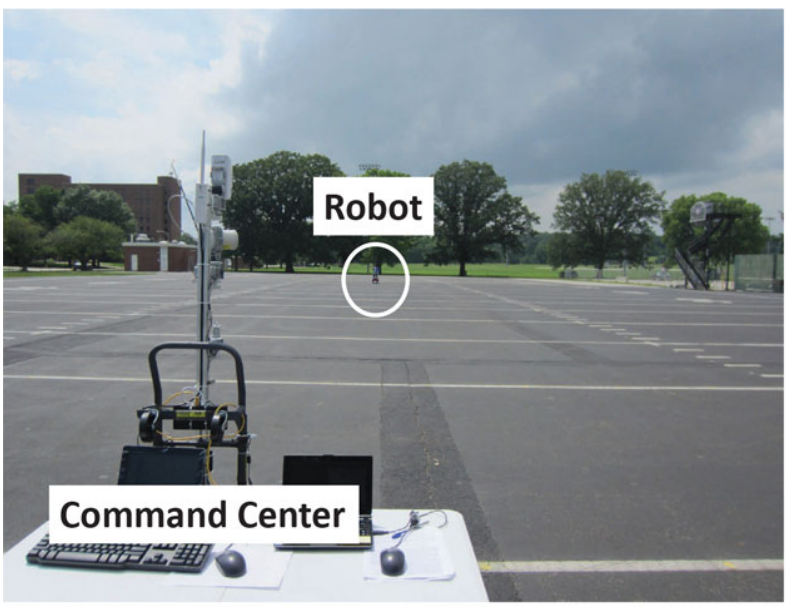

(a)

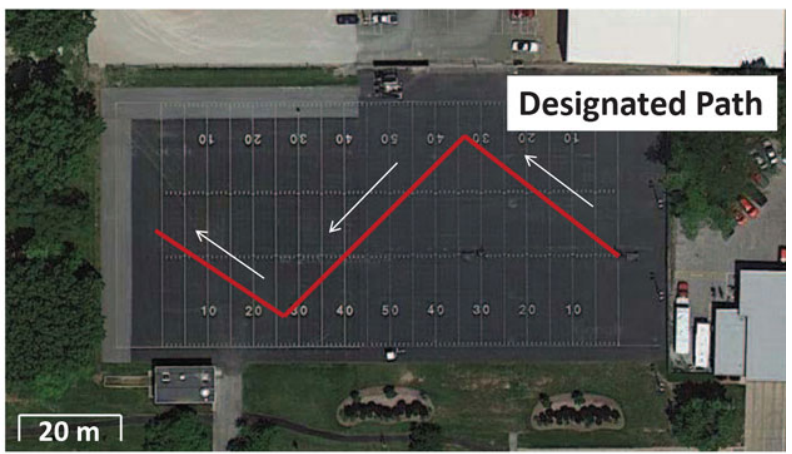

(b)

Figure 13. Outdoor test in open environments at Purdue main campus. (a) Purdue marching band practice field. (b) Designated path.

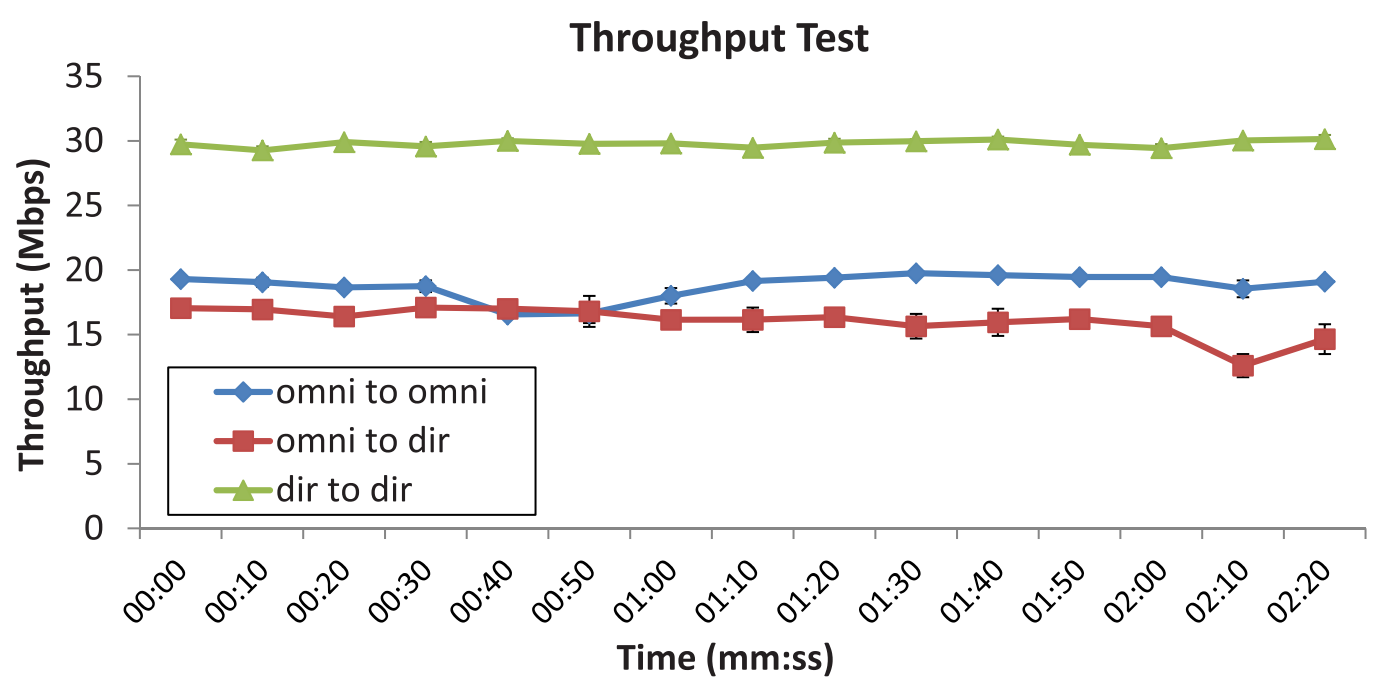

Figure 14. Throughput measured while the robot was moving in an outdoor and open environment.

To support this conclusion, we show the results of the estimated DOA by the bottom antennas on each side in Figure 15. In this figure, the estimated DOA by the robot's antenna is depicted with a red arrow, and the estimated DOA by the command center's antenna is depicted with a blue dotted arrow. They are all averaged over three trials and projected on the designated paths by considering positions and poses of the robot. Consequently, the arrows by the robot's estimation and the arrows by the center's estimation formed almost a straight line on most of the locations except when the robot turned a corner. This indicates that our proposed system can enable proper antenna tracking, and therefore optimize the orientations of the two top anten- nas without acquiring the physical orientation and location of the antenna.

According to Dabin, Haimovich, \& Grebel (2006), the second selection would have less path loss than the first selection, therefore resulting in better throughput performance. However, from Figure 14, the second selection showed slightly poorer performance as the robot moved farther away from the command center. Conversely, the first selection showed stable performance over all the distances and times. Overall, the second selection showed poorer performance than the first selection in this test. Actually, this result was quite different from what we have expected. We are not sure yet, but this unexpected result could come 


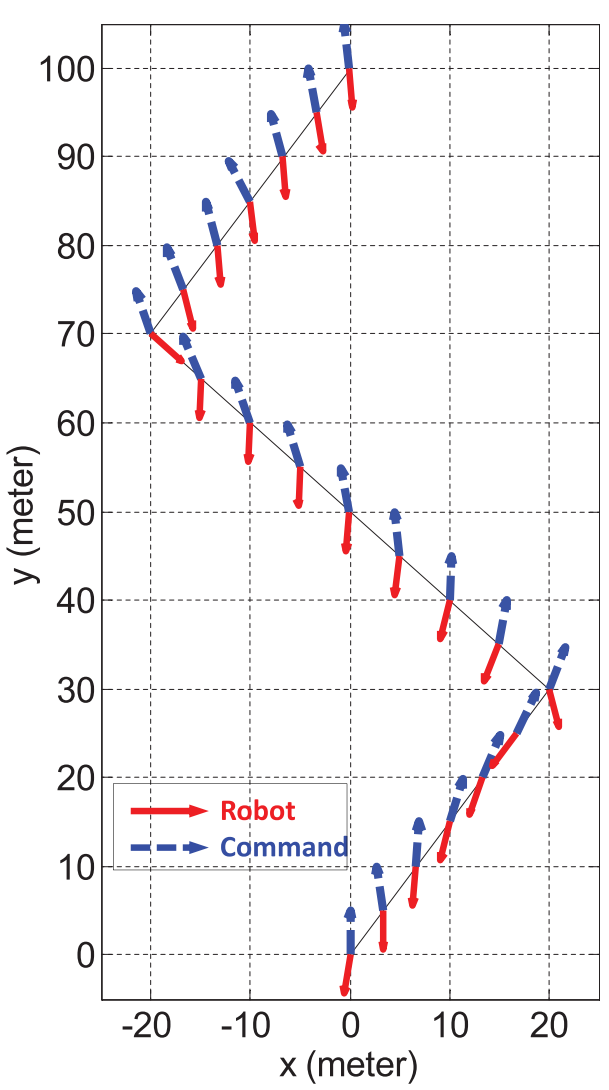

Figure 15. Estimated DOA with the bottom antennas on the robot side and the command center side.

from the effect on wireless network channel conflicts with other wireless devices, which can significantly slow all the involved networks. In fact, this test area is located right next to college dormitories, and thus there are a lot of private APs that are actively operated. As we mentioned earlier in Section 2.2, it would be possible that omnidirectional antennas used in the first and second antenna selections experienced interference from other radio signals.

\subsection{Outdoor Test in Complex Environments}

Next, to observe quantitative differences in throughput when the robot is moving from a line-of-sight region to a non-line-of-sight region, we conducted experiments at the Lafayette fire training center as shown in Figure 16. Because the facility is completely free from private APs, this test was also especially designed to investigate effects on wireless interference that we speculated about from the previous experiment in Section 5.2.

For this test, we initially placed the robot $15 \mathrm{~m}$ away from the command center and made it move from a lineof-sight region to a non-line-of-sight region with an almost constant speed of $0.1 \mathrm{~m} / \mathrm{s}$ as shown in Figure 16(c). More specifically, this environment provided a line-of-sight between the robot and the command center until the robot moves to $4 \mathrm{~m}$ from the initial location. And then, as the robot moves forward, the line-of-sight region disappears and the level of the non-line-of-sight region between the robot and command center fades in. This environment setting is depicted in Figure 16(c). As shown in this figure, there is non-line-of sight between the robot and the command center once the robot moves farther than $4 \mathrm{~m}$.

Figure 17 shows throughput results from all the tests with each antenna selection. For experiments with a fair evaluation, each setting was run through three trials, and Figure 17 shows the averaged throughput with standard deviation represented with an error bar. As shown in Figure 17, the third selection, directional-to-directional antennas dominantly outperforms the other two, especially in a non-lineof-sight region. Specifically, when the robot was equipped with the first two antenna selections, a wireless connection could not be maintained at all after moving farther than $7 \mathrm{~m}$ and $9 \mathrm{~m}$, respectively. The first antenna selection, omni-to-omni antennas, fails to compromise in situations where line-of-sight is unavailable as throughput drops at 7 Mbps per $1 \mathrm{~m}$ away from a line-of-sight region. The second antenna selection, omni-to-directional antennas, struggled but was able to maintain a wireless connection at a farther distance from the initial location compared to the first antenna selection. There is a peak throughput measured when the robot was at $8 \mathrm{~m}$ from the initial location, and it was apparent that reflected RF signals were able to converge well and thus throughput could be increased. When the robot was equipped with the third antenna selection, throughput was maintained at high values (and, indeed, had few differences) until the robot reached $13 \mathrm{~m}$, which the robot could not reach when only equipped with the first two antenna selections. In addition, throughput had a small decrease until the robot reached the end of the testing area. In fact, the robot could have moved to much further distances, but due to the limited space of the environment, we had to stop at $15 \mathrm{~m}$. Considering the final configuration, where one antenna attached on the robot points in the same direction as the other antenna attached at the command center, this result could be expected.

Since there was no wireless interference from private APs at this location, we were also able to investigate our speculation regarding the previous test where the first antenna selection showed a better performance than the second selection due to wireless interference. As shown in Figure 17, the second antenna selection had a noticeably higher throughput than the first selection in all locations. Moreover, the second and third antenna selections show almost the same throughput results in a line-of-sight region. To sum up, we were able to confirm our speculation regarding the unexpected results that arises from surrounding wireless interference and validated the expected order of throughput in ideal cases, which was as follows: 


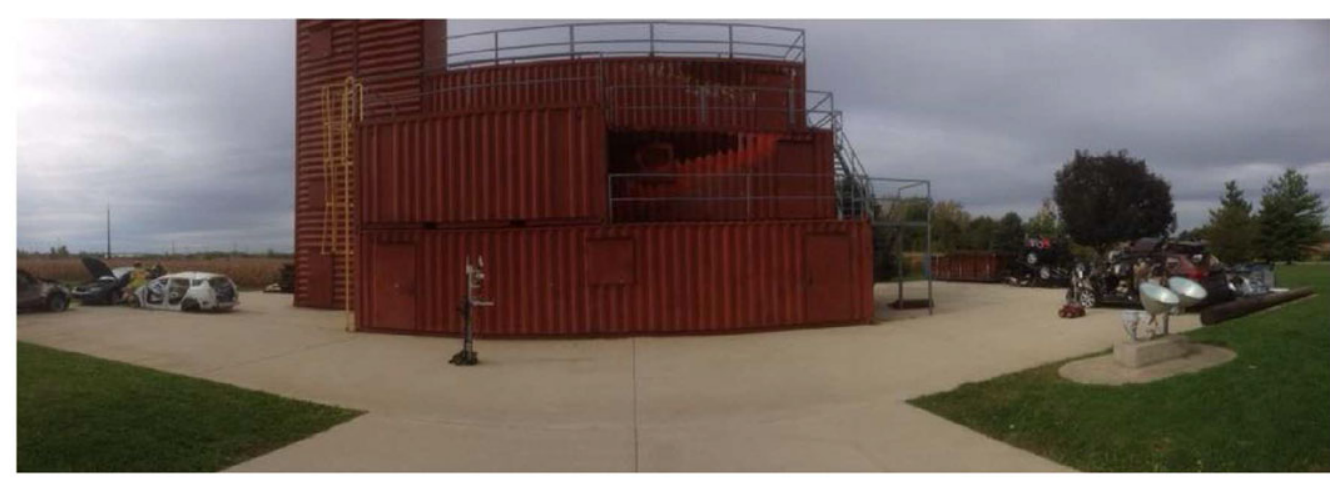

(a)

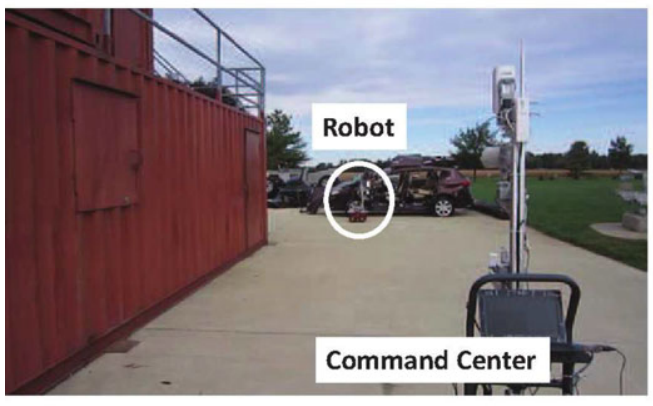

(b)

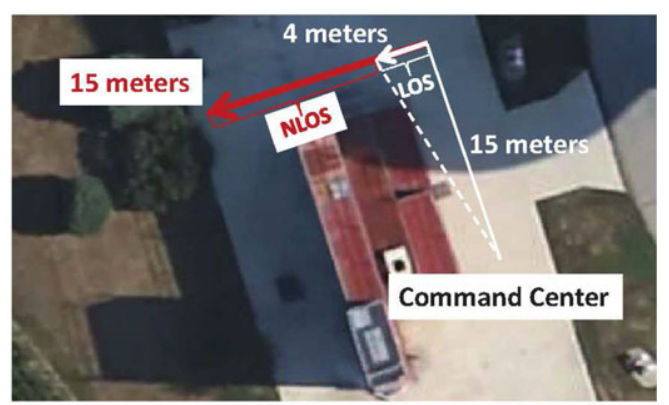

(c)

Figure 16. Outdoor test in a complex and wireless interference-free environment at the Lafayette fire training center, Indiana, USA. (a) Fire training facility. (b) Initial location. (c) Experiment settings.

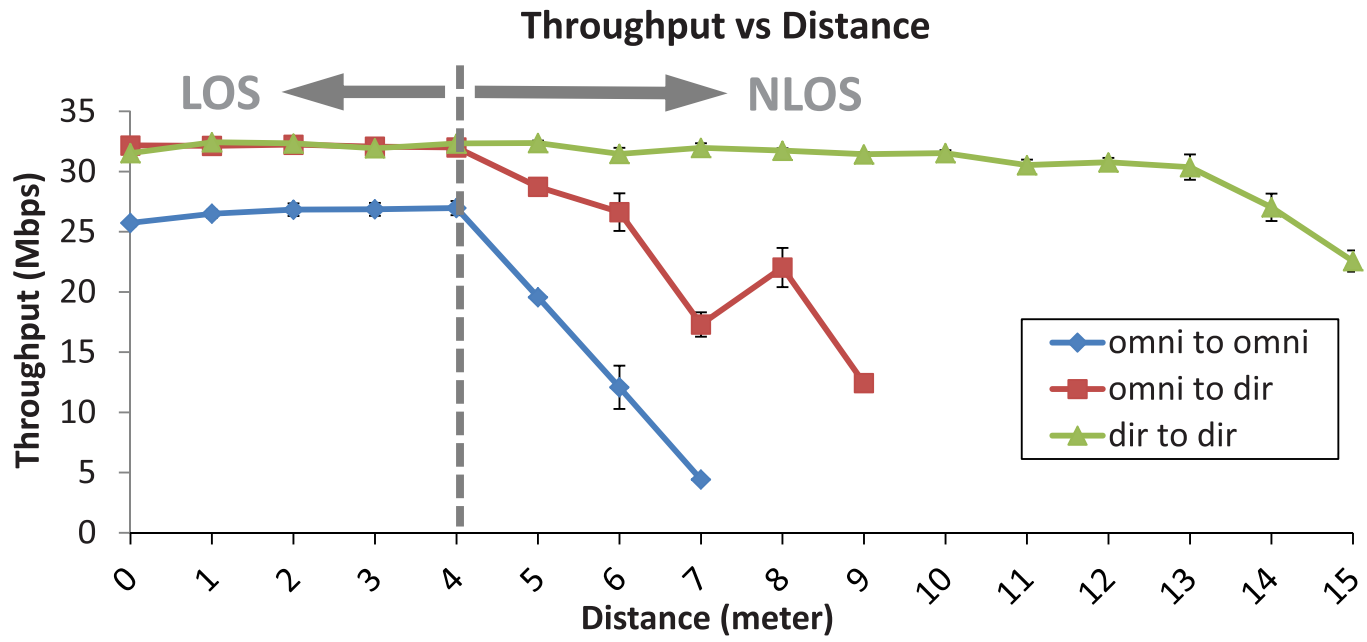

Figure 17. Throughput measured while the robot was moving from a line-of-sight region (LOS) to a non-line-of-sight (NLOS) region and there was no wireless interference from private APs.

1) directional-to-directional antenna (best), 2) omni-todirectional antenna, and 3) omni-to-omni antenna (worst).

\subsection{Indoor Test}

It is known that the use of directional antennas is inappropriate in indoor environments in general. However, this type of antenna would be helpful in near line-of-sight coverage such as long hallways or corridors. For this reason, we chose a hallway of Knoy Hall at Purdue University for the third environment and tested our proposed system, as shown in Figure 18(a). This test was specifically designed to test cases where the robot needs to be deployed inside buildings. 


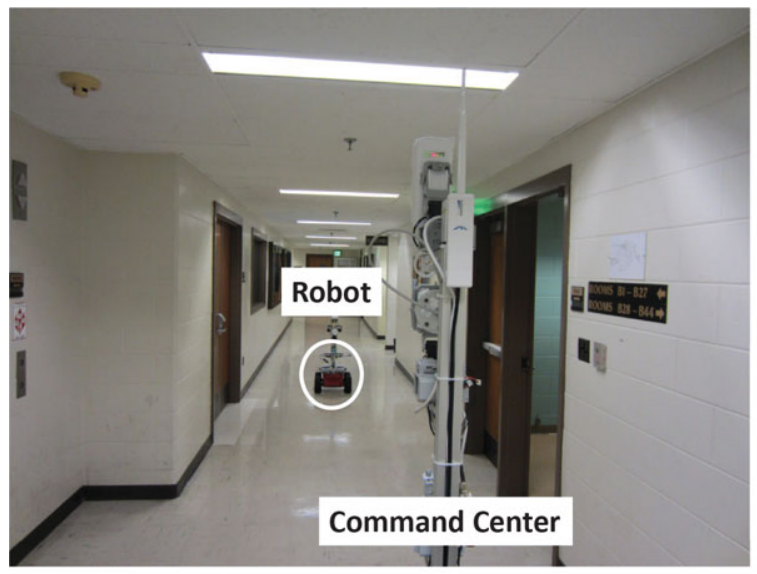

(a)

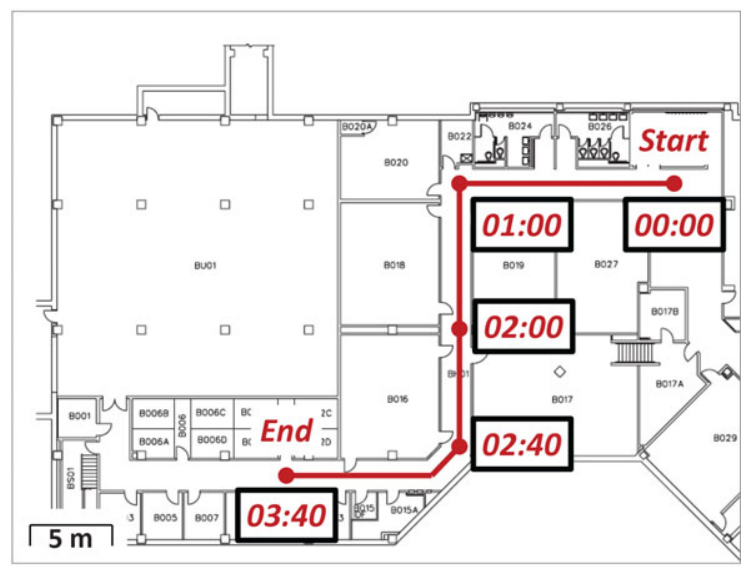

(b)

Figure 18. Indoor test. (a) Purdue Knoy hall. (b) Designated path.

During this test, the robot and the command center were initially collocated at 00:00, as indicated in Figure 18(b). The robot was then set to move along a designated path with an almost constant speed of $0.2 \mathrm{~m} / \mathrm{s}$. The designated path is shown in Figure 18(b). The total traveling distance of this path is approximately $50 \mathrm{~m}$, so it takes about $4 \mathrm{~min}$ to reach the final location. As shown in the floor map in Figure 18(b), the robot was supposed to experience various situations, including line-of-sight and non-line-of-sight. Hence, this environment was good to check our antenna tracking system in more detail.

This experiment was repeated three times, and Figure 19 shows the averaged estimated DOA with standard deviation represented with an error bar. DOA was estimated by the bottom antenna on the robot side over the total travel. First, estimated DOA remained around $0^{\circ}$ until the robot approached the first corner (see time from 00:00 to 01:00). As soon as the robot started turning counterclockwise, estimated DOA increased to positive values until the robot's pose crossed at right angles to the command center. Then, as the robot started moving forward again, estimated DOA went to around $0^{\circ}$, and decreased to negative values, reaching to a $-30^{\circ}$ angle. In fact, these negative values result from the geometry of the environment. That is, because the directional antenna on the command center faced toward the front view for most of the time, its radio signal was reflected by the left wall and the upper wall around the first corner as if the original signal source was from that spot. To receive this reflected radio, the antenna on the robot side had to face in the left direction, resulting in negative values in DOA estimation. This result persisted until the robot entered the middle of the path. Then, when the robot turned clockwise at the second corner, the directional antenna oriented to the left direction, resulting in negative values in DOA estimation. This estimation persisted until the robot reached the final location. From this analysis on the history of estimated
DOA, we could validate that our proposed antenna tracking system works properly.

Figure 20 shows throughput measured while the robot was moving from the initial location to the final location. Unlike the previous two experiments, all three antenna selections showed almost the same performance until the robot reached the middle of the designated path. Even, the third selection showed the lowest throughput until the robot entered non-line-of-sight regions (see around time of 00:50). As omnidirectional antennas are known to perform well in indoor environments, this result could be expected. However, as the robot moved farther from the command center, specifically after 02:00, throughput results between the first selection and the other two showed a noticeable gap. That is, the second and third selections employing directional antennas showed significantly better performance than the first selection even in an indoor environment.

\section{CONCLUSIONS}

Reliable distant wireless communication between multiple robots in the network-free environment is one of the most important and unsolved issues, especially for the firefighting-like multiple rescue robot systems. For high reliability of those kinds of distant wireless robot communication, one of the most important problems is to maintain strong connectivity between robots or between a robot and a command center. Through this paper, we have suggested more reliable networked robotic system based on a specially configured robot with directional antennas and the proposed WCA-based active antenna tracking system. By the proof of convergence of WCA-based active antenna tracking algorithm and by various experiments in various real fields, we have confirmed the feasibility and effectiveness of our proposed robotic configuration and our antenna tracking system. We have also showed that 


\section{DOA Estimated on Robot}

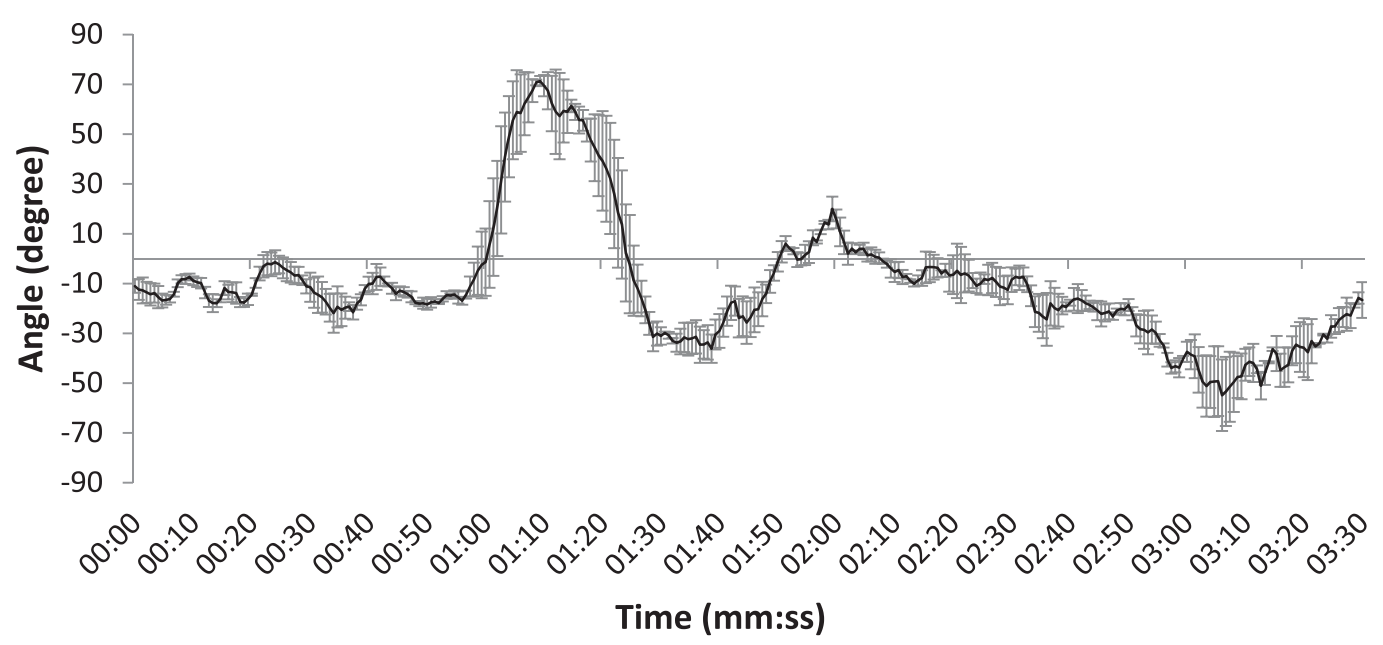

Figure 19. Estimated DOA with the bottom antenna on the robot side.

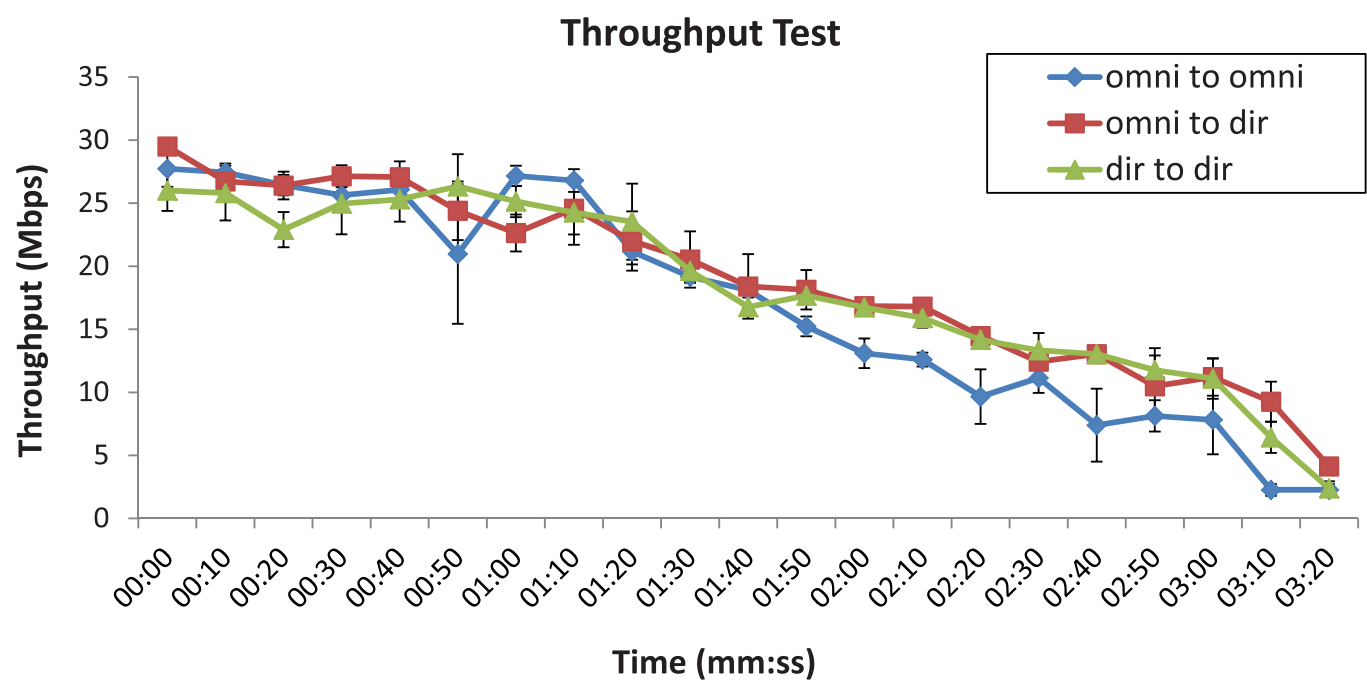

Figure 20. Throughput measured while the robot was moving in an indoor and complex environment.

our directional-to-directional antenna structure for robot communication is much better in terms of maintaining strong network connectivity, compared with omni-to-omni or omni-to-directional one.

More specifically, through field tests in outdoor and open environments, the configuration consisting of the directional-to-directional antennas showed far higher throughput by as much as one and a half times. In the setting where the robot moved from a line-of-sight region to a non-line-of-sight region, the configuration with directionalto-directional antennas dominantly outperformed the other two. The configuration showed far higher throughput and was also able to maintain strong wireless connectivity in a non-line-of-sight region. From these results, we conclude that the third selection is applicable to non-line-of-sight regions as well. Furthermore, from indoor tests, we verified that directional antennas can be used and show satisfactory performance in indoor environments. We do need to also acknowledge that the environment where we conducted indoor experiments may confer some advantages for the operation of directional antennas because the environment is composed of long and narrow corridors where RF radio can be better propagated by using a directional beam rather than an omnidirectional beam. Therefore, this novel design of a robotic communication system can also be used for a wide variety of robotic applications, from rescue robots to military robots, and from security robots to space robots. 
In future work, we will verify our system in much longer and larger spaces to make it more robust and to cope with Fresnel zone issues that were not taken into account in this paper. To cope with those issues, for instance, we could consider exploiting an elevation (tilt) angle in the directional antennas. Also, we will devise a new pan-tilt device allowing for a greater azimuthal pointing range for the directional antennas in order to further maximize the performance of our system.

\section{REFERENCES}

Bapna, D., Rollins, E., Foessel, A., \& Whittaker, R. (1998). Antenna pointing for high bandwidth communications from mobile robots. In Robotics and Automation, 1998. Proceedings. 1998 IEEE International Conference on (Vol. 4, pp. 3468-3473).

Behnke, R. \& Timmermann, D. (2008). AWCL: adaptive weighted centroid localization as an efficient improvement of coarse grained localization. In 5th Workshop on Positioning, Navigation and Communication, 2008. WPNC 2008 (pp. 243-250).

Blumenthal, J., Grossmann, R., Golatowski, F., \& Timmermann, D. (2007). Weighted centroid localization in ZigBee-based sensor networks. In Intelligent Signal Processing, 2007. WISP 2007. IEEE International Symposium on (pp. 1-6).

Dabin, J. A., Haimovich, A., \& Grebel, H. (2006). A statistical ultra-wideband indoor channel model and the effects of antenna directivity on path loss and multipath propagation. Selected Areas in Communications, IEEE Journal on, 24(4), 752-758.

Dai, H.-N., Ng, K.-W., Li, M., \& Wu, M.-Y. (2013). An overview of using directional antennas in wireless networks. International Journal of Communication Systems, 26(4), 413448.

Elnahrawy, E., Austen-Francisco, J., \& Martin, R. (2007). Adding angle of arrival modality to basic rss location management techniques. In Wireless Pervasive Computing, 2007. ISWPC '07. 2nd International Symposium on.

Everett, E., Duarte, M., Dick, C., \& Sabharwal, A. (2011). Empowering full-duplex wireless communication by exploiting directional diversity. In Signals, Systems and Computers (ASILOMAR), 2011 Conference Record of the Forty Fifth Asilomar Conference on (pp. 2002-2006).

Graefenstein, J., Albert, A., Biber, P., \& Schilling, A. (2009). Wireless node localization based on RSSI using a rotating antenna on a mobile robot. In 6th Workshop on Positioning, Navigation and Communication, 2009. WPNC 2009 (pp. 253-259).

Hood, B.\& Barooah, P. (2011). Estimating DoA from radiofrequency RSSI measurements using an actuated reflector. IEEE Sensors Journal, 11(2), 413-417.

Hsieh, M. A., Cowley, A., Kumar, V., \& Taylor, C. J. (2008). Maintaining network connectivity and performance in robot teams. Journal of Field Robotics, 25(1-2), 111-131.

Kim, M.\& Chong, N. Y. (2007). RFID-based mobile robot guidance to a stationary target. Mechatronics, 17(4-5), 217-229.
Kim, M.\& Chong, N. Y. (2008). Direction sensing RFID reader for mobile robot navigation. IEEE Transactions on Automation Science and Engineering, 6(1), 44-54.

Lee, J.-S., Su, Y.-W., \& Shen, C.-C. (2007). A comparative study of wireless protocols: Bluetooth, UWB, ZigBee, and Wi-Fi. In Industrial Electronics Society, 2007. IECON 2007. 33rd Annual Conference of the IEEE (pp. 46-51).

Mahmood, A., Javaid, N., \& Razzaq, S. (2015). A review of wireless communications for smart grid. Renewable and Sustainable Energy Reviews, 41, 248-260.

Min, B.-C., Lewis, J., Matson, E. T., \& Smith, A. H. (2013a). Heuristic optimization techniques for self-orientation of directional antennas in long-distance point-to-point broadband networks. Ad Hoc Networks, 11(8), 2252-2263.

Min, B.-C., Matson, E., \& Khaday, B. (2013b). Design of a networked robotic system capable of enhancing wireless communication capabilities. In Safety, Security, and Rescue Robotics (SSRR), 2013 IEEE International Symposium on (pp. 1-8).

Nguyen, C. Q., Min, B. C., Matson, E. T., Smith, A. H., Dietz, J. E., \& Kim, D. (2012). Using mobile robots to establish mobile wireless mesh networks and increase network throughput. International Journal of Distributed Sensor Networks, 2012.

Pezeshkian, N., Nguyen, H. G., \& Burmeister, A. (2007). Unmanned ground vehicle radio relay deployment system for non-line-of-sight operations. In Proceedings of the 13th IASTED International Conference on Robotics and Applications, RA '07 (pp. 501-506).

Pivato, P., Palopoli, L., \& Petri, D. (2011). Accuracy of RSS-based centroid localization algorithms in an indoor environment. IEEE Transactions on Instrumentation and Measurement, 60(10), 3451-3460.

Sayrafian-Pour, K.\& Kaspar, D. (2006). Application of beamforming in wireless location estimation. EURASIP Journal on Advances in Signal Processing, 2006, 158-158.

Shahzad, K. \& Oelmann, B. (2014). A comparative study of insensor processing vs. raw data transmission using ZigBee, BLE and Wi-Fi for data intensive monitoring applications. In Wireless Communications Systems (ISWCS), 2014 11th International Symposium on (pp. 519-524).

Tekdas, O., Kumar, Y., Isler, V., \& Janardan, R. (2012). Building a communication bridge with mobile hubs. IEEE Transactions on Automation Science and Engineering, 9(1), 171176.

Tuna, G., Gungor, V. C., \& Gulez, K. (2014). An autonomous wireless sensor network deployment system using mobile robots for human existence detection in case of disasters. Ad Hoc Networks, 13, 54-68.

Wang, J., Urriza, P., Han, Y., \& Cabric, D. (2011). Weighted centroid localization algorithm: Theoretical analysis and distributed implementation. IEEE Transactions on Wireless Communications, 10(10), 3403-3413.

Yi, S., Pei, Y., \& Kalyanaraman, S. (2003). On the capacity improvement of ad hoc wireless networks using directional antennas. In Proceedings of the 4th ACM international symposium on Mobile ad hoc networking \& computing (pp. 108-116). ACM. 